\title{
Colonization and Bacteremia due to Peripheral Venous Catheters in Hospitalized Children
}

\author{
Gholamreza Soleimani ${ }^{1}$, Shahram Shahraki Zahedani ${ }^{2}$, Seyed Hosein Soleimanzadeh Mousavi ${ }^{3}$, \\ Ali Soleimani ${ }^{4}$, Motahareh Parsa ${ }^{5}$, Vida Veysi Bigzadi ${ }^{6}$ and Elham Shafighi Shahri*7 \\ ${ }^{1}$ Associate Professor of Pediatric infectious disease, Children and Adolescents Research Center, Iran \\ ${ }^{2}$ Associate Professor of Microbiology, Infectious Diseases and Tropical Medicine Research Center, Iran
}

${ }^{3}$ Department of Pediatrics, School of Medicine, Ali-Ibn-Abitaleb Hospital, Iran

${ }^{4}$ MD, FRACGP, Casey Super clinic, Australia

${ }^{5}$ MD, FRACGP, Seymour Medical Clinic, Australia

${ }^{6} \mathrm{MD}$, Zahedan University of Medical Sciences, Iran

${ }^{7}$ Assistant Professor of Pediatrics, Zahedan University of Medical Sciences, Zahedan, Iran

*Corresponding author: Elham Shafighi Shahri, Assistant Professor of Pediatric infectious disease, Children and Adolescents Research Center, Zahedan, Iran

\section{ARTICLE INFO \\ Received: 幽 February 09, 2021 \\ Published: 幽 February 18, 2021 \\ Citation: Gholamreza Soleimani, Shahram Shahraki Zahedani, Seyed Hosein Soleimanzadeh Mousavi, Ali Soleimani, et al. Colonization and Bacteremia due to Peripheral Venous Catheters in Hospitalized Children. Biomed J Sci \& Tech Res 34(1)-2021. BJSTR. MS.ID.005493.}

Keywords: Colonization; Bacteremia; Peripheral venous catheters; Intensive care unit

\section{ABSTRACT}

Introduction: Intravenous injection is one of the important principles in the treatment of most patients, which has complications such as catheter site infection that it is necessary to identify the factors that increase the prevalence of venous catheter infections. Therefore, the aim of this study was to evaluate the frequency of colonization and bacteremia caused by peripheral venous catheters in hospitalized children.

Materials and Methods: This cross-sectional study was performed on 70 children with venous catheter. After recording demographic information, colonization and bacteremia caused by peripheral venous catheters in patients, Chi-square and MannWhitney tests were used to compare data.

Results: The results of the present study showed that the frequency of bacterial colonization in peripheral catheters in children was $22.9 \%$ which was not related to the demographic characteristics of patients and their underlying disease history $(\mathrm{P}<0.05)$. The most common cultured bacterium was Staphylococcus epidermidis.

Conclusion: Considering the prevalence of colonization in peripheral catheters in children, it is recommended that the necessary training be provided to the staff of this department and preventive measures be taken in high-risk individuals to reduce nosocomial infections and complications.

\section{Introduction}

Although 70 years have passed since the life of intravenous therapy, the belief in injecting drugs into the bloodstream has undoubtedly been a human dream for centuries [1]. Today, more than $90 \%$ of hospitalized patients are treated intravenously during their treatment [2]. and over 500 million peripheral venous catheters are placed annually [3,4]. According to a study conducted in Yazd, 50\% [5] and a similar study in Tehran, 55\% of hospitalized patients undergo intravenous treatment that found the extent of the use of this method of treatment in the country [4]. Unfortunately, this route of drug prescription is not without its drawbacks and has many side effects which the most common is heart disease and the most dangerous is septicemia [5]. Intravenous catheters are associated with a variety of infectious complications, from local infection of the catheter site to Catheter-related bloodstream infection (CRBSI), endocarditis, and other metastatic infections [6]. 
Catheter infections increase patients' morbidity, prolong hospitalization, and increase mortality [7]. It is estimated that more than 200,000 cases of CRBSI occur annually in the United States, resulting in approximately $12-25 \%$ mortality [8]. CRBSI incidence relate to the catheter type, the catheter usage prolongation, manipulation of it and patient's background diseases [9]. The most common central venous catheter used in patients is the no tunneled CVC and this catheter is responsible for $90 \%$ of all systemic catheter infections. Catheter insertion into a jugular vein increases the risk of bacteremia. This is due to contamination of the catheter dressing caused by contact with oral discharge. Catheter-induced bacteremia is the most serious complication of venous catheter use [10]. Although the infection may be due to contamination of the injectable material, or colonization of the catheter hub, most infections are due to the secondary implantation of the patient's dermal flora microorganisms into the intradermal pathway of the catheter $[6,9,11]$. Have been shown a correlation between isolated organisms in bacteremia caused by catheter and the skin flora adjacent to catheter [10-12].

Since the source of many infections caused by the catheter is the bacterial invasion of the skin flora adjacent to the catheter, observing the principles of sterilization and the use of topical antiseptic agents during implant placement and in subsequent catheter care can theoretically be considered as one Catheter infection prevention methods. The use of topical antiseptic compounds such as alcohol, betadine and especially chlorhexidine during catheter insertion and dressing change is recommended to prevent infections caused by central venous catheter. Since fibrin deposition in the catheter can be the primary nucleus of microbial colonization, the use of anticoagulants to prevent colonization and catheter infection has been suggested [13]. As mentioned, the prevalence of catheter infection and its complications is high and depends on different factors that are different in each region and country, so recognizing the prevalence, influencing factors and symptoms and its prognosis in the treatment of patients and measures what is done is effective. Therefore, the aim of this study was to investigate the prevalence of colonization of venous catheters and the resulting bacteremia in the intensive care unit of Ali Ibn Abitaleb Hospital.

\section{Methods}

This descriptive-analytical study was performed to investigate the prevalence of colonization of venous catheters and the resulting bacteremia admitted to pediatric wards and intensive care units of
Ali-Ibn-Abitaleb Hospital in 2018-2019. In this study, all children with peripheral venous catheter whose peripheral venous catheter was removed were included in the study. Pre-designed questionnaire that includes demographic characteristics (sex, age, time of hospitalization, disease, history of infection). Clinical findings (swelling, discoloration, low pain), information about the location of the catheter (neck, hands, feet, head), The time elapsed since the venipuncture (less than 48 hours and more than 48 hours) and the conditions during the venipuncture, the type of medication used, the presence or absence of complete venous nutrition, and the cause of the catheter (hemodialysis fluid intake, central pressure measurement) filled.

Then, after removing the catheter from its fixed area in the body, we cut the last $5 \mathrm{~cm}$ into a sterile way and gave it to the laboratory under sterile conditions. At the same time, we took a blood sample from the patient and sent it to the same laboratory for culture. During all this time, we informed the study participants and their families about this study. All patient's information including demographic factors, clinical and paraclinical symptoms were recorded in a checklist and entered SPSS software, and finally statistical analysis was presented in two descriptive and analytical sections.

\section{Results}

The aim of this study was to investigate the frequency of colonization and bacteremia caused by peripheral venous catheters in children admitted to pediatric wards and intensive care units and related causes in Ali-Ibn-Abitaleb Hospital in Zahedan in 2018-2019. This study was performed on 70 children, of which $43(61.4 \%)$ were boys and 27 (38.6\%) were girls. The results of our study showed that the mean age of patients was $4.14 \pm 3.92$ years. Out of 70 children included in the study, the prevalence of colonization and bacteremia due to peripheral venous catheters was 16 (22.9\%). Also, out of 70 patients studied, 9 (12.9\%) had positive blood culture. The most common cultured mass was Staphylococcus epidermidis (50\%) and then E. coli (25\%). The most common bacteria in blood culture were Staphylococcus epidermidis (55.6\%). (Table 1) The mean and standard deviation of age in children with and without positive bacterial culture were not statistically significant different. $(\mathrm{P}=0.081)$ There was no statistically significant difference in prevalence of sex in children with and without positive bacterial culture. $(\mathrm{P}>0.05)$.

Table 1: Frequency of cultured bacteria from peripheral venous catheters and blood culture in patients with positive colonization.

\begin{tabular}{|c|c|c|c|c|}
\hline & Bacteria & Frequency & Percentage & The cumulative percentage \\
\hline \multirow{5}{*}{ Peripheral venous catheters } & E. Coli & 4 & 25 & 25 \\
\hline & Staphylococcus epidermidis & 8 & 50 & 75 \\
\hline & Enterobacteriaceae & 2 & 12.5 & 87.5 \\
\hline & staphylococcus aureus & 2 & 12.5 & 100 \\
\hline & All & 16 & 100 & - \\
\hline
\end{tabular}




\begin{tabular}{|c|c|c|c|c|}
\hline \multirow{4}{*}{ Blood culture } & non hemolytic streptococcus & 1 & 11.1 & 11.1 \\
\cline { 2 - 5 } & Staphylococcus epidermidis & 5 & 55.6 & 66.7 \\
\cline { 2 - 5 } & E. coli & 1 & 11.1 & 77.8 \\
\cline { 2 - 5 } & Pseudomonas & 1 & 11.1 & 88.9 \\
\cline { 2 - 5 } & Micrococcus & 9 & 11.1 & 100 \\
\cline { 2 - 5 }
\end{tabular}

In only 4 of the patients whose test was positive, an underlying disease was reported, of which 2 had heart disease, one had diabetes, and one had another disease. These statistics and Table 2 show that there was no significant difference in the frequency and type of underlying diseases in patients. After investigating the cause of hospitalization, the most common causes were gastroenteritis and febrile convulsion (16 patients) followed by pneumonia (6 patients). However, according to the performed analyzes (P-Value=0.412), no significant difference was observed in the cause of hospitalization of patients in both positive and negative cultures. Similarly, no significant differences were observed in the length of hospitalization of patients in both groups. (P-Value=0.989).

Table 2: Abundance of underlying disease in children with and without positive bacterial culture.

\begin{tabular}{|c|c|c|c|c|c|c|}
\hline Underlying Dis & & & Negative Culture & Positive Culture & All & P-Value \\
\hline \multirow{4}{*}{ No Underlying Disease } & \multirow{2}{*}{ No } & Num. & 42 & 12 & 54 & \multirow{4}{*}{1} \\
\hline & & Percentage & 77.8 & 22.2 & 100 & \\
\hline & \multirow{2}{*}{ Yes } & Num. & 12 & 4 & 16 & \\
\hline & & Percentage & 75 & 25 & 100 & \\
\hline \multirow{8}{*}{ Underlying disease } & \multirow{2}{*}{ Cardiac } & Num. & 7 & 2 & 9 & \multirow{8}{*}{0.631} \\
\hline & & Percentage & 77.8 & 22.2 & 100 & \\
\hline & \multirow{2}{*}{ Renal } & Num. & 3 & 0 & 3 & \\
\hline & & Percentage & 100 & 0 & 100 & \\
\hline & \multirow{2}{*}{ Diabetes } & Num. & 1 & 1 & 2 & \\
\hline & & Percentage & 50 & 50 & 100 & \\
\hline & \multirow{2}{*}{ Other } & Num. & 1 & 1 & 2 & \\
\hline & & Percentage & 50 & 50 & 100 & \\
\hline
\end{tabular}

\section{Discussion}

Out of 70 children included in the study, the prevalence of colonization and bacteremia due to peripheral venous catheters was $16(22.9 \%)$. Also, out of 70 patients studied, 9 (12.9\%) had positive blood culture. The most common cultured organism was Staphylococcus epidermidis (50\%) and then E. Coli (25\%). The most common organism in blood culture was Staphylococcus epidermidis (55.6\%). In a study conducted by Daneshi et al. For the prevalence of colonization of venous catheters and the resulting bacteremia in the neonatal intensive care unit of Qods Hospital in Qazvin (2005) 100 infants admitted to the NICU who had their catheter removed for any reason were examined. $3 \mathrm{~cm}$ tip of each catheter was cut under sterile conditions and inserted into thioglycollate culture media. Up to 7 days later, if any bacterial colonies were observed on the culture media, the colonies were cultured on blood agar and McConkey media and their identity and antibiogram were determined. A blood sample was taken from each of these children between the catheter and before the catheter was removed for culture and microbiological study. The results of this study showed that $35 \%$ of 100 cultured catheters were colonized and 19 cases (52\%) were staphylococcal coagulase negative. Seven of the blood cultures (77) were positive (4 cases of coagulasenegative staphylococci and 3 coagulase-positive staphylococci), all of which were related to catheter [14]. Although the prevalence of positive cases in this study was twice that of our study, but like our study, staphylococcal coagulase was the most common. The reason for the prevalence of positive cases may be due to differences in the sample size, differences in patients' demographic indicators, differences in inclusion and exclusion criteria, and differences in how patients are sampled.

In a study conducted by Nick Farid et al. to investigate the relationship between the shelf life of peripheral venous catheter and the incidence of venipuncture complications. The results of the present study showed that the prevalence of peripheral venous catheter colonization was $33.6 \%$ and the most common organism was coagulase negative staphylococcus ( $50 \%$ positive). Colonization was less in the group receiving intravenous antibiotics and more in the group receiving anticonvulsant drugs than the other groups. On the other hand, there was no significant relationship between colonization and local complications of catheterization. In the end, 
it was concluded that increasing the shelf life of peripheral venous catheter from 48 hours to 72 hours does not increase the risk of catheterization complications such as phlebitis and subcutaneous fluid leakage [15]. Although the frequency of positive cases in this study is 1.5 times than of our study, but similar to our study, staphylococcus coagulase negative was the most common.

In a study conducted by Pourbazaz et al. With the aim of infections related to intravascular catheters in children in 2002, it was stated that the infection rate and colonization of arterial and venous catheters are almost similar. For umbilical artery catheter infection in infants with very low birth weight (VLBW) and use of antibiotics for more than 10 days, and for umbilical vein catheter, more birth weight and use of intravenous feeding fluids as risk factors Are known. Regardless of the type of catheter, the most common organism responsible for circulatory infection is coagulase-negative staphylococcus [16]. The results of this study are in line with the findings of our study. In a study by Patricia et al. of the 391 short-term peripheral venous catheters, $27.7 \%$ of the catheter tip and $11.3 \%$ of the catheter canals were colonized by bacteria. Phlebitis was observed in $17.4 \%$. Staphylococcus aureus (5.60\%), Staphylococcus epidermidis (23.5\%) were common bacteria isolated from the cloned catheter tip. The most common organism isolated from the catheter canal was Staphylococcus aureus (56.8\%) and then Staphylococcus epidermidis (18.1\%) [17]. The prevalence of positive cases in the study was twice that of our study, and the most common was not like our study.

In a study by Jeffrey et al., phlebitis, extravasation, and bacterial colonization occurred at $13 \%, 28 \%$, and $11 \%$, respectively. Catheters were often colonized with coagulase-negative staphylococci (54.5\%). Catheter placement time ( $>144$ hours, odds ratio 5.8) was an important determinant of catheter colonization. Diazepam injections (odds ratio 11.0) or fat emulsions (odds ratio 2.5) and age ( $\geq 1$ year, odds ratio 5.8) were also important risk factors for colonization [18]. The prevalence obtained in this study is like our study. Contrary to this study, in our study, there was no difference in the incidence of colonization based on age, type of prescription and other factors.

\section{Conclusion}

The results of the present study showed that the frequency of bacterial colonization in peripheral catheters in children was equal to $22.9 \%$ which was not related to the demographic characteristics of patients and their underlying disease history. Our study also found that the most common cultured bacterium was Staph epidermis. Therefore, due to the prevalence of colonization in peripheral catheters in children, it is recommended that the training the staff of this department and preventive measures be taken in high-risk individuals to reduce nosocomial infections and reduce complications.

\section{Acknowledgements}

The authors thank the emergency medical service personnel, Pediatric Residents, nurses, and emergency physicians who participated in the study. This article was supported by the Children and Adolescents Health Research Centre-Zahedan.

\section{Funding}

This study was supported by nowhere.

\section{Declaration of Interest}

The authors report no conflicts of interest. The authors alone are responsible for the content and writing of this article.

\section{References}

1. Jayaweera JAAS, Sivakumar D (2020) Asymptomatic central lineassociated bloodstream infections in children implanted with long term indwelling central venous catheters in a teaching hospital, Sri Lanka. BMC Infect Dis 20: 457.

2. Kendirli T, Yaman A, Ödek C, Özdemir H, Adem K, et al. (2017) Central line-associated bloodstream infections in pediatric intensive care unit. J Pediatr Emerg Intensive Care Med 4(2): 42-46.

3. Kothalawala M, Jayaweera JAAS, Arunan S, Jayathilake JAMA (2019) The emergence of non-albicans candidemia and evaluation of HiChrome Candida differential agar and VITEK2 YST® platform for differentiation of Candida bloodstream isolates in teaching hospital Kandy, Sri Lanka. BMC Microbiology 19(1): 136.

4. (2020) Bloodstream Infection Event (Central Line-Associated Bloodstream Infection and Non-central Line Associated Bloodstream Infection). CDC. Device associated module.

5. Ray-Barruel G, Xu H, Marsh N, Cooke M, Rickard CM (2019) Effectiveness of insertion and maintenance bundles in preventing peripheral intravenous catheter-related complications and bloodstream infection in hospital patients: a systematic review. Infect Dis Health 24(3): 152168.

6. Flynn J, Zhang Y, Solar-Yohay S, Shi V (2012) Clinical and demographic characteristics of children with hypertension. Hypertension $60(4)$ : 1047-1054.

7. Jackson SS, Leekha S, Magder LS, Pineles L, Anderson DJ, et al. (2017) The effect of adding comorbidities to current centers for disease control and prevention central-line-associated bloodstream infection riskadjustment methodology. Infect Control Hosp Epidemiol 38(9): 1-6.

8. Miller DL, O'Grady NP (2003) Guidelines for the prevention of intravascular catheter related infections: recommendations relevant to interventional radiology. J Vasc Interv Radiol 1419(2): S355-358.

9. Konstantinidi A, Sokou R, Panagiotounakou P, Lampridou M, Parastatidou S, et al. (2019) Umbilical Venous Catheters and Peripherally Inserted Central Catheters: Are They Equally Safe in VLBW Infants? A NonRandomized Single Center Study. Medicina 55(8): 442.

10. Pérez-Granda MJ, Bouza E, Pinilla B, Cruces R, González A, et al. (2020) Randomized clinical trial analyzing maintenance of peripheral venous catheters in an internal medicine unit: Heparin vs. saline. PLoS ONE 15(1): 226-251.

11. Zhong L, Wang HL, Xu B, Yuan Y, Wang X, et al. (2017) Normal saline versus heparin for patency of central venous catheters in adult patients-a systematic review and meta-analysis. Crit Care 21(1): 5.

12. You T, Jiang J, Chen J, Xu W, Xiang L, et al. (2017) Necessity of heparin for maintaining peripheral venous catheters: A systematic review and meta-analysis. Exp Ther Med 14(2): 1675-1684. 
13. Abdelkefi A, Torjman L, Ladeb S, Othman TB, Achour W, et al. (2005) Randomized trial of prevention of catheterrelated bloodstream infection by continuous infusion of low dose unfractionated heparin in patients with hematologic and oncologic disease. J Clin Oncol 23(31): 7864-7870.

14. Daneshi M, Sarookhani M, Habibi M (2007) Colonization rate of intravascular catheters and catheter related bacteremia in neonatal intensive care unit of Qods Hospital, Qazvin (2005). J Qazvin Univ Med Sci 11(3): 9-13.

15. Nikfarid L, Khogasteh N, Ghanbarian A (2006) Investigation of relation between dwell time of peripherally intravenous catheters in hospitalized neonates and children. Hayat 12(1): 17-25.

\section{ISSN: 2574-1241}

DOI: 10.26717/BJSTR.2021.34.005493

Elham Shafighi Shahri. Biomed J Sci \& Tech Res

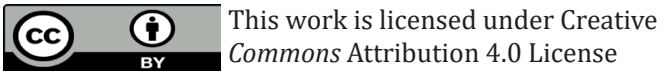

Submission Link: https://biomedres.us/submit-manuscript.php
16. Ismail Pourbzaz Negin (2002) Intravascular catheter-related infections in pediatric patients Prevention and treatment. Iranian Children's Diseases Journal 13: 14-20.

17. Patricia Nahirya, Justus Byarugaba, Sarah Kiguli, Deogratias KadduMulindwa (2008) Intravascular catheter related infections in children admitted on the paediatric wards of Mulago hospital, Uganda. Afr Health Sci 8(4): 206-216.

18. Jeffery S Garland, Peter Havens, W Michael Dunne, Mary Hintermeyer, Mary Anne Bozzette, et al. (1992) Peripheral Intravenous Catheter Complications in Critically III Children: A Prospective Study. Pediatrics 89(6): 1145-1150.

$\begin{array}{ll}\text { BIOMEDICAL } & \text { Assets of Publishing with us } \\ \text { RESEARCHES } & \text { - Global archiving of articles } \\ \text { - Immediate, unrestricted online access }\end{array}$

